\title{
Construction of Intelligent Logistics Warehouse Management Information System based on RFID Technology
}

\author{
ShuMin Duan * \\ College of Tourism \\ Kaifeng University \\ Henan Kaifeng, 475000, China \\ duanshuminedu@163.com \\ * Corresponding Author
}

\author{
Yan $\mathrm{Xu}$ \\ College of Computer and Information Engineering \\ Anyang Institute of Technology \\ Henan Anyang, 455000, China
}

\begin{abstract}
In this paper, the characteristics and functions of radio frequency identification (RFID) technology are analyzed. Then it discusses the method of constructing the intelligent logistics warehouse management information system. RFID system is applied to the management of goods in the warehouse. It can not only deal with the storage of goods, warehouse and inventory management, but also can supervise all the goods. The paper presents construction of intelligent logistics warehouse management information system based on RFID Technology. The proposed method can effectively save labor costs, improve the accuracy of the work, and ensure the quality of the product.
\end{abstract}

Keywords- Radio Frequency Identification (RFID); Intelligent Logistics; Warehouse Management; Inventory Management; Electronic Tags

\section{INTRODUCTION}

Electronic tags, also known as radio frequency tags, transponder, and it is generally by the coupling element (antenna) and the special chip. Electronic tags are the real data carriers for RFID systems, each with a unique electronic encoding (ID), and the tag generally holds the electronic data in the agreed format [1]. In practical applications, RFID tags are usually attached to different types, different shapes of objects surface, and even embedded into the object, so it will be based on the need to make different shapes.

Traditional warehousing and logistics in a large extent need to spend a lot of manpower, material resources, now the use of RFID technology can be very good management of goods in and out of the warehouse in all aspects, such as: goods into the library, classification, distribution of shelves, such as a library number, name of commodity, specifications, origin, distribution, manufacturers and other details. And it can be very easy to get the information in the most convenient way. Logistics is now widespread use of bar code technology, although the degree of intelligent than before, but still need to spend a lot of manpower and material resources into the storage and logistics.

The reader is responsible for reading and writing the label information of the device, the basic function of the reader is to provide a way to carry out data transmission with the radio frequency tags. The typical reader includes a control unit, a radio frequency transceiver module, a read write antenna and an interface module. The controlling unit can handle the input and output data of the radio frequency chip according to the data integrity, the encoding rule, the encryption and decryption protocol, and to determine whether the data is reasonable.

RFID in logistics management, the advantages of RFID technology is a process control technology, can provide a strategic significance for the supply chain of manufacturing, logistics and wholesale and retail industry. RFID technology can help companies increase the amount of information exchange, speed up the flow of information, and thus improve efficiency, save costs. In recent years, RFID technology has so much attention.

Storage is an indispensable part in logistics, which plays an important role in the whole logistics process. Storage is a buffer pool of raw materials, semi-finished products and finished products in order to provide support for the continuous and stable production [2]. At the same time, storage can overcome the time and space differences between producers and consumers, support enterprise logistics strategy, improve customer service levels, and reduce logistics costs. It can be said to leave the warehouse support, it is impossible to have a high efficiency and low cost of logistics services.

The logistics industry is expected to implement the "RFID" strategy, which is based on the technology of wireless radio frequency identification technology, which is implemented by WAL-MART, a large retailer in the west, to further improve efficiency, create new profit growth point, and seek a greater competitive advantage over its competitors. The new application of the Internet of things in the modern logistics, logistics industry is bound to have an important and profound impact on the logistics warehousing management, supply chain management, distribution system intelligence, logistics information and so on. The paper presents construction of intelligent logistics warehouse management information system based on RFID Technology. 


\section{INTELLIGENT EXPLORATION BASED ON RADIO FREQUENCY IDENTIFICATION TECHNOLOGY}

Devices that read (and sometimes write) label information may be designed for handheld or fixed; the reader can read and identify the electronic data stored in the electronic tag without touching, thereby achieving the purpose of automatic identification of objects. Usually the reader is connected to a computer, and the read label information is transmitted to the computer for further processing.

RFID is the abbreviation of Frequency Identification Radio, that is, radio frequency identification technology, commonly known as electronic tags. It is a non-contact automatic identification technology, which is composed of computer, reader, antenna, electronic tags. Controlled by computer, automatic identification of signals, antennas emit electromagnetic waves, radiation corresponding range of electronic tags, and then the reverse direction of the transmission signal, and finally to the computer, the identification work without manual intervention, can work in a variety of harsh environment.

An interface module that is attached to the reader supports RS232/485 or Ethernet, so that the data is transmitted to a data management system or from a management system receiving command [3]. The reader can be fixed or handheld; in general, the handheld reader because the transmission power is limited, so the communication distance is relatively short. In the case of Save, it is fixed at the end of the read write distance of 300 feet $(100 \mathrm{~m})$ while holding the read and write head of the read and write distance of 200 feet (about 60m).

The rapid development of RFID design and manufacturing technology, the price of RFID tags will be greatly reduced, and the last obstacle to the wide application of RFID will be cleared. Early investment in RFID technology will have a great advantage in its competitors, which drives most companies to study, develop and use the new technology as soon as possible. RFID enabled the company to obtain huge profits, mainly including reduced inventory and sales personnel costs, reduce read code labor costs and inventory savings, reduce theft and out of stock etc.

RFID there is no uniform standards for the current industry standards and there are not unified, the electronic standard signed so far the world has not yet formally formed some unified international standards. The standard is not uniform is the important factor restricting the development of RFID, the standard is not unified also so that the current RFID products are not compatible with each other, which is bound to hinder the future development of RFID products. At present, there are two kinds of encoding system for the application of RFID in logistics.

$$
I^{\prime}=H(k \mid k-1)-H(k \mid k)
$$

Wireless radio frequency identification (RFID) is a kind of automatic recognition technology that can identify target objects and obtain relevant information without manual operation, and can be used in various kinds of harsh environments. This technology has been widely used in the field of material flow.
Unique intelligent fault processing technology, in the terminal equipment failure automatic alerts and processing, and even auto repair, and does not affect the normal work of the other terminal equipment, greatly reduce the maintenance difficulty, unique open equipment management technology, about different network terminal equipment were independent and convenient to add, configuration and management, even control equipment acquisition speed and working state, as is shown by equation (2) [4].

$$
J_{m}=\left\|I^{*} \psi_{\mu, v}(z)\right\|
$$

According to the different electronic tags can be divided into read and write card (RW), a time to read the card (WORM) and read only card (RO). RW cards are generally more expensive than WORM and RO cards, such as phone cards, credit cards, WORM card is the user can write a one-time card, write data can not change, the $\mathrm{RW}$ card to be cheaper; RO card has a unique number, can not change, to ensure the security.

The typical application mode of RFID in supply chain is the tracking application of logistics. In the technical realization, the RFID tag can be attached to the tray, the packing box or the components, and the components and the serial number and the information of the automatic storage and transmission. The RFID tag is able to transmit the information to a certain range of readers, so that the warehouse and the workshop no longer need to use a handheld barcode reader to read and write the components and finished one by one scan bar code. This can reduce the occurrence of the omission in a certain extent, greatly improve the working efficiency [5]. The application model can significantly cut costs and clean up the obstacles in the supply chain, as is shown by equation (3).

$$
x_{1}=a, x_{2}=x_{1}+h, x_{3}=x_{2}+h=a+2 \Delta x
$$

RFID usually consists of three parts: (1) tag (tag): composed of coupled components and chips, attached to identify the target object in the object, each tag has only electronic coding; (2) readers (Reader): read the label information of equipment; (3) antenna: between the tag and the reader transmit radio frequency signals.

RFID RF card, due to the use of wireless communication, in the reader's effective communication range, there may be a number of tags (radio frequency card), which requires the reader to have the ability to prevent collision. When there are more than one RFID RF card readers in the work of the card reader's antenna, reader's anti collision module of the reader will be launched. Under the control of an anti collision handling program, the card reader will first communicate with each card and obtain the label number of each card (UID). Since each RFID RF card has its unique.

\section{ANALYSIS ON THE CONSTRUCTION OF INTELLIGENT WAREHOUSE LOGISTICS MANAGEMENT SYSTEM}

The existing logistics, the majority of the use of bar code as a warehouse management intelligent way, although the degree of intelligent than before, but still need 
to spend a lot of manpower and material resources into the storage and logistics. The main operation process of modern logistics is the distribution of the vehicle will require the delivery of the goods to the warehouse area and in accordance with the pre customized good location, using the "advanced first out" principle to carry out the storage area.

Port logistics process mainly includes the typical export business, import business and logistics scheduling. By the shipper to the freight forwarding commissioned; freight forwarding to shipping booking $\mathrm{B}$ when shipping booking confirmation; freight forwarding to customs clearance D. when customs inspection after the release; the freight forwarding to the terminal for set port formalities finally; shipper packing set Hong G. Container import business. First the shipping to on behalf of the goods arrival notice; then by the forwarder shipping to return in exchange for delivery records; by the forwarder and customs clearance; after waiting for customs inspection release; the freight forwarding to the station for suitcase procedures [6].

$$
a(\theta)=\left[\begin{array}{c}
1 \\
\exp (j \pi u) \\
\vdots \\
\exp (j(M-1) \pi u)
\end{array}\right]^{T}
$$

Transport links in the transportation management area, through the transport of goods and vehicles is to paste RFID tags (such as the label affixed to the container and equipment) to achieve tracking control equipment. RFID reading and writing device is usually installed in the transport line some check points (such as post or next to the pier, etc.) and storehouses, stations, docks, airports and other key locations. The receiving device receives the RFID tag information, and transmits the position information of the location to the communication satellite, and then transmitted to the transportation dispatching center input data base by the satellite.

Logistics RFID system is still in the pilot phase has revealed a huge development potential. And in the United States Ship2Save company has officially opened its use of RFID system of intelligent warehouse facilities. This is North America's most advanced automation equipment warehouse. The goods to the warehouse can be recorded by the active and passive RFID tags. These devices enable not only to monitor the amount of inventory, but also to distinguish between the various goods and to detect the movement of goods. If the goods are not recorded, the facility will be taken out of the alarm.

Warehouse management plays a key role in logistics management. The traditional warehousing industry is to take charge for the business model, and I hope my warehouse is always full, this model and the purpose of the logistics runs counter. Modern logistics in order to integrate the process, coordination on the lower reaches of its responsibility, the more the better the static inventory; the business model is also built on the total cost of logistics assessment. Because these two types of warehouse management in the business model has an essential difference, but in the specific operation such as storage, warehousing, sorting, tally and it is difficult to distinguish, so on the analysis and research must pay attention to the similarities and differences between them, these similarities and differences will be reflected in the structure of the information system.

User management is to have the right to use the system of the basic situation of the user data to update, query, etc. to achieve user management functions. User level management and it has different permissions, and has the function of group management users [7]. Material management is the type of all materials in the warehouse (including name, factory, etc.) to update, delete and query operations, to achieve material management functions Materials can be dynamically added to support batch management, support for multi measurement unit.

$$
<f_{i}>_{t}=\frac{\int_{-\infty}^{\infty} V(t, f) f d f}{\int_{-\infty}^{\infty} V(t, f) d f}
$$

In the final payment of the customer, just push the right products through the RFID reader, you can see the amount of their own on the computer screen directly, rather than spend a long time waiting for the cashier to use a scanner to scan a piece of goods after the payment. This saves consumers time for a single query, but also improve the work efficiency of retailers.

When the goods are in stock, and it is to each inventory management of electronic labels, and it is the label for the goods labels. Posted tagged items should be easy to install, FCL or entire pallet label items. In warehouse operation, read the number of tags, determine whether the work is correct. In order to save operating costs, the goods labels are designed to be reusable [8]. When the goods are taken out of the warehouse, and then sent to the warehouse to re use. Set up a wireless network, covering the entire warehouse operations area, all of the operating data in real time transmission. Install the fixed wireless data terminal on the forklift truck, hand in hand with the portable wireless data terminal. The wireless data terminal has the function of receiving the operation instruction, confirming the location of the job and whether the operation is accurate and returning the job.

Automatically create shipping manifest and automatic shipping notice (ASN) accurate transport of goods - in the right time and place, adjust the transport of goods, to correct goods instead of the extra goods to reduce their goods damage and deterioration of chance - the use of warehouse management system (WMS) triggered automatically remove the product warning through the record label on the effective date.

\section{CONSTRUCTION OF INTELLIGENT LOGISTICS WAREHOUSE MANAGEMENT INFORMATION SYSTEM BASED ON RFID TECHNOLOGY}

Warehouse management system is a real-time computer software system, it can operate according to the business rules and algorithms (algorithms), the information, resources, behavior, inventory and distribution operations 
more perfect management, so that it can maximize meet the requirements of effective output and accuracy. The "warehouse" referred to here includes all types of storage and distribution centers in the fields of production and supply, and of course, including general warehouses, logistics warehouses and freight forwarders.

The application method of RFID identification system in container management is usually, the label is pasted, embedded in a container or a tray, the reading and writing device of the entrance of the reader or the reader or handheld reader to identify the label of the dynamic information, read the information transmitted to the monitor or database in real time [9]. Container RFID recognition system can simultaneously identify 40 pallets and 80 plastic containers, as is shown by equation (6).

$$
O C=\sum_{t=0}^{T}\left(\sum_{j=1}^{n+1} \sum_{k=1}^{n+1} c_{j}(t) c_{k}(t) / x^{2}(t)\right)
$$

In addition to the cost of RFID, in addition to the previously mentioned above, the other problem is the price of electronic tags [10]. This is also an important aspect of RFID application. As with office supplies, because the storage is a large quantity of goods for storage, if each item is labeled and each label is a lot of money, it will also greatly improve the cost of warehousing. Only the price of electronic tags down, it is possible to order and use of RFID in bulk, otherwise, the high cost of electronic tags brought about by the cost, not every manufacturer has to bear.

Intelligent warehouse management system is composed of central data server and management terminal. It is the data center of the system. It is responsible for communicating with the handset, converting the data of the handheld computer and inserting it into the database.

The unique "data analysis" technology can analyze the data of various manufacturers and models, support data packet length analysis, identification analysis and comprehensive analysis, the "professional data" into "common data"; powerful "multi task concurrent processing" technology, can support more than 500 terminal equipment at the same time, "standardized data output" technology, according to user needs to convert data into decimal, sixteen, string format, easy data statistics and analysis.

RFID can play a key role in many aspects of logistics, including retail, storage, transportation and distribution. RFID retail sectors through effective tracking inventory and transportation, can improve the retailer's inventory management, to achieve timely replenishment, improve efficiency, to reduce the error [11]. At the same time, radio frequency tags can be used to monitor and control the quality of the goods; the store can use the RFID system to achieve automatic scanning and billing, in order to replace the low efficiency of the manual collection.

The warehouse receipt operator uses the wireless mobile device to scan the material bar code specified in the application form, and enter the number of the input. If the material is provided with a tray, the bar code is scanned. From the warehouse management system to check the availability of the scanned material information, select the storage location of the material, if it is stored in the database of the material needs to be tested, it is to enter the number of material inspection. After the warehouse operator scans the collection application form the specified material is submitted to the warehouse management system for the corresponding processing, the submitted information including material bar code, the actual collection quantity, the tray bar code and the library bar code, and specify the number of materials in each library, after the submission of the collection operation.

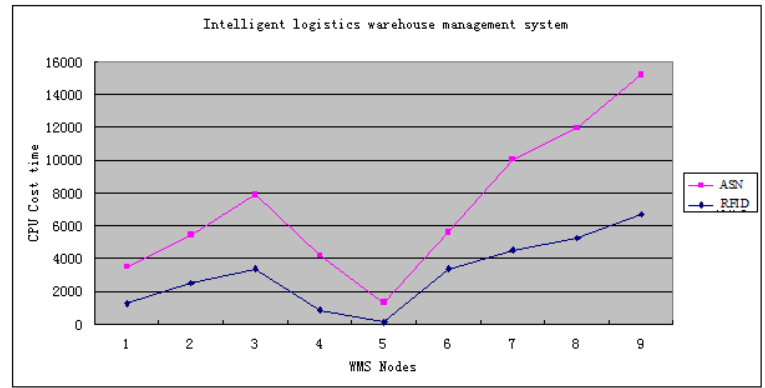

Figure 1. Comparison of Intelligent Logistics Warehouse Management Information System Based on RFID and ASN.

Work area: can track the transport documents with RFID tags in real time and automatically print the transport documents, and update the truck, and send the automatic shipping notification (ASN). Inventory levels: the internal and future supply chain operation of asset management permanent identification of the goods and other containers to reduce processing and dwell time; enhance the visibility of assets, improve asset utilization, reduce the safety stock, increase the assets to help reduce the fixed assets on hand After the optimization of the above work, the full use of the characteristics of RFID technology, and it is from many aspects of the work to improve the efficiency and quality of the purpose, to achieve the expected goal.

\section{SUMMARY}

The paper presents construction of intelligent logistics warehouse management information system based on RFID Technology. It is suggested that the warehouse logistics center consider its own situation and the external environment, as soon as possible to improve the efficiency and quality of the work, to enhance the management and improve the service of the target, to enhance the enterprise image, expand the business area, will have a profound impact. At the same time, also hope that more manufacturers will be as soon as possible research and development of RFID products, and promote the rapid development of the logistics industry.

\section{REFERENCES}

[1] Jee - Hee Koo, Tae - Woong Jung, Ji - Yeon Ahn, Sang - Rak Lee, "Development of Livestock Management Module using RFID for Livestock Farms", RNIS, Volume 14, pp. $711 \sim 714,2013$

[2] $\mathrm{Xu} \mathrm{Xu}, \mathrm{Liu}$ Wei, "Research on Logistics Distribution Center Location Model Based On GIS", JDCTA, Vol. 7, No. 7, pp. 538 545, 2013.

[3] Feng Feng, Xu Qi, "Locating Method in the Warehouse Based on Active RFID Tags", JDCTA, Vol. 7, No. 1, pp. 223 231, 2013.

[4] Shufang Li, "Research on Customer Satisfaction of Logistics Outsourcing Based on AHP: An Example from Manufacturing Enterprises of China", AISS, Vol. 5, No. 3, pp. $668 \sim 675,2013$. 
[5] JIAO YABING, "Construction and Application of Logistics Information Tracking System Based on RFID Technology", JCIT, Vol. 8, No. 10, pp. $837 \sim 845,2013$

[6] Yonghui Zhang, Xinhua Jiang, Zhangxi Lin, Quan Liang, "PreHandover Context Transfer Mechanism Based on Warehouse Station Communication Pattern", JDCTA, Vol. 6, No. 19, pp. 206 $\sim 214,2012$.

[7] Kwang Namgung, Min Soo Kang, Sun Shin Ahn, "A Study on the Common Standard Platform for Logistics Information Sharing on RFID and Barcode", JNIT, Vol. 4, No. 6, pp. 1 8, 2013.

[8] Peihua Fu, Xiaoli Gu, Qiang Wang, "Fuzzy Clustering Analysis of Goods Classification Based on Equivalence Relation in Inventory Management", IJACT, Vol. 5, No. 4, pp. 507 514, 2013.
[9] DOU Xue-xia, "The Building of University Assets Information Management System based on RFID", IJACT, Vol. 5, No. 2, pp. $289 \sim 297,2013$

[10] Zhanying Zhang, Ziping Zhao, "A Logistics Information Platform Based on J2EE Lightweight Framework", JDCTA, Vol. 7, No. 9 , pp. $395 \sim 401,2013$

[11] Wei Jiang, Yingcheng Xu, Yuexiang Yang, Wenqiong Li, "An Improved Genetic Algorithm for Optimization of Two-Echelon Water Resources Inventory Management", AISS, Vol. 5, No. 8, pp. $796 \sim 805,2013$. 